\title{
DIABETES STATUS IS ASSOCIATED WITH POOR COGNITIVE PERFORMACE IN SAUDI POPULATION AT HIGH METABOLIC RISK
}

\author{
T. Alaama ${ }^{1,2}$, M. Basheikh ${ }^{1,2}$, A. Khiyami ${ }^{1}$, M. Mutwalli ${ }^{1}$, S. Batawi ${ }^{1}$, G. Watfa ${ }^{3}$
}

\begin{abstract}
Objective: Previous studies have shown that Diabetes mellitus (DM) is associated with an increased risk of cognitive impairment, but little data is available on Arabic populations, inspite of their remarkably high prevalence of DM. In this study we attempt to study the effect of DM on cognitive performance in middle-aged and elderly patients. Design: Observational cross sectional study. Setting: Outpatient clinics in King Abdulaziz University Hospital (KAUH) in Jeddah, Saudi Arabia. Participants: The study included 241 volunteers aged $59.6 \pm 9.2$ years; 171 outpatients with DM, matched with 70 controls without. Measurements: Volunteers underwent cognitive assessment using the Montreal Cognitive Assessment Test (MoCA) and the Rowland Universal Dementia Assessment Scale (RUDAS). Results: RUDAS score was poorer in diabetics (25,25 \pm 2,78 vs. 26,71 $\pm 2,57$ in controls; $\mathrm{p}<0.0001$ ) who are more likely to have cognitive impairment $16 \%$, than those who are not diabetics $3 \%$; $\mathrm{p}=0.004$. This association was confirmed in multivariate analyses and shown to be independent of female gender and low education level, all of which were associated with worse RUDAS cognitive score.The results were not significant when the MoCA was used, as $85 \%$ of the cases and $78 \%$ of the controls had abnormal results; $\mathrm{p}=0.194$. Among diabetics, there was no statistically significant effect found for glycemic control or DM duration on either one of the tests. The prevalence of obesity was similar in the two groups with $63 \%$ in diabetics and $62 \%$ in controls. Conclusion: In our population with an alarming prevalence of obesity, diabetes was associated with poorer cognitive performance independent of female gender or low education level, drawing attention to this under-recognized problem of cognitive impairment that could result in significant health and social problems, particularly in areas with high diabetes prevalence. RUDAS was found to be a very reasonable and convenient test to assess cognition in our sample characterized by a low educational level.
\end{abstract}

Key words: Diabetes mellitus (DM), cognitive impairment, RUDAS, MoCA, Kingdom of Saudi Arabia (KSA).

\section{Introduction}

Diabetes Mellitus (DM) is a prevalent condition worldwide. However, the prevalence of DM in Saudi Arabia is alarmingly high, ranging from 18 to $30 \%(1,2)$.

Moreover, the International Diabetes Federation (IDF) identified 6 countries of the Middle East and North Africa, including Saudi Arabia, among the world's ten highest countries in the prevalence of DM (3).

Previous studies have shown that diabetes is associated with an increased risk of cognitive impairment and the development of dementia $(4,5)$.

As life expectancy improves in Saudi Arabia (6), the Saudi elderly population is expected to grow resulting in unmasking of more of these age-related complications in

1. King Abdulaziz University, Jeddah, Kingdom of Saudi Arabia (KSA); 2. King Fahad Medical Research Center, Jeddah, Kingdom of Saudi Arabia (KSA); 3. King Saud Medical City, Community Health, Riyadh, Kingdom of Saudi Arabia (KSA)

Corresponding Author: Dr. Ghassan WATFA, MD; PhD, King Saud Medical City Community Health Centre, 12746 Riyadh, Kingdom of Saudi Arabia (KSA), Tel: +966599192881; E-mail: ghassanwatfa@hotmail.com

Received June 7, 2016

Accepted for publication July 6, 2016 the future.

However, most of the existing studies addressing relationship between diabetes and cognitive impairment have been conducted in industrial countries, while few or no data is available in emerging populations and Arabic populations. Moreover, the majority of studies were conducted in the elderly population, however it has been suggested that cognitive decline could begin in midlife in diabetic patients (7).

This study attempts to assess in a tertiary hospital-King Abdulaziz University Hospital (KAUH) in Jeddah, Saudi Arabia, the cognitive performance in middle-aged and elderly diabetic patients with comparison to matched controls.

\section{Patients and methods}

A cross sectional study was carried out from June to July 2012 in the outpatient department of King Abdulaziz University Hospital (KAUH), Jeddah, Kingdom of Saudi Arabia. This study included a total of 171 outpatients 
with diabetes, matched with 68 controls without.

The studied patients were consecutively recruited among patients who were approached while they waited for appointments at various hospital clinics. Data collection took place in the Internal medicine clinics.

Participants were included if they were aged $\geq 45$ years and had signed an informed consent form. Subjects were excluded if they had documented dementia or in the case of absence of a hospital medical record number.

Volunteers underwent cognitive assessment using two validated tests: the Montreal Cognitive Assessment Test (MoCA), and the Rowland Universal Dementia Assessment Scale (RUDAS).

The Montreal Cognitive Assessment is a brief cognitive screening tool. A score of 30 is obtained when all answers are correct. The MoCA has been found to have a specificity of $87 \%$ and a sensitivity of $90-100 \%$ for detecting mild cognitive impairment (MCI) with a cutoff score of 26 ( 25 or below indicating impairment) (8).

The RUDAS is a six item screening test scored out of a total of 30 points. It assesses language, praxis, memory, judgment, construction and fluency. Performance on RUDAS has been shown to be affected by age, but not by education, gender or preferred language. The RUDAS dementia screening cut-off is a score of $<23$ which yields a specificity of $95.8 \%$ and a sensitivity of $81 \%$ (9).

Cognitive assessments were carried out by researchers who were trained by experienced geriatricians prior to the study period. Training of researchers took place over several weeks; through sessions in which the tests were explained and performed several times to ensure interrater and intra-rater reliability.

As well as undergoing the MoCA and the RUDAS, the Geriatric Depression Scale (GDS) (10) was administered to all subjects and several other variables were collected. These included: age, gender, and marital status, smoking habits, level of education, body mass index (BMI), waist circumference (WC), number of comorbidities and history of hypertension, depression, coronary heart disease (CHD), and stroke or transient ischemic attack (TIA). For diabetic patients, $\mathrm{HbA} 1 \mathrm{C}$ results and the duration of diabetes were also collected.

Regarding marital status, subjects were categorized into two groups, married and others (single, window or divorced). Patients were classified into two categories according to smoking habits as never smokers and past smokers or current smokers. Education level was classified into four categories according the years of education: illiterate ( $<1$ year), Fundamental (1-6 years), intermediate ( $>6-12$ years) and Higher Education $(>12$ years).

Anthropometric measurements were recorded for each subject. Patients' weight in kilograms and height in meters were both measured using a Seca Medical Physician Electronic Scale. BMI was then calculated according to the equation: $\mathrm{BMI}=$ Weight $(\mathrm{kg}) / \mathrm{Height}^{2}$ $\left(\mathrm{M}^{2}\right)$. The WC was measured for each subject at the umbilicus directly after expiration. Results were recorded in centimeters.

\section{Statistical Analysis}

Sample size considerations were based on RUDAS score values established in diabetic patients (11) and in general population previously studies (12), a minimum of 63 diabetic patients and 63 controls was considered as necessary for an alpha risk of $5 \%$ and a beta risk of 20 $\%$.Since our population could be different from the above mentioned studies, it was therefore decided to include more subjects than the minimum indicated above.

The data was analyzed using NCSS 9 statistical software package (Kaysville, UT). Descriptive values are expressed as means \pm SD or percentages. The two-tailed significance level was set to $p=0.05$.

The variables were compared using Student T-test, Chi-Square tests or ANOVA, as appropriate. Pearson's correlation test was performed to examine various correlations.

Analyses of factors associated with RUDAS were performed using multiple regression analyses with an interactive backward selection method. Validity of the model assumption was verified using analysis of model residuals and testing for heteroscedasticity.

\section{Results}

\section{Study population characteristics}

The present study encompassed 241 participants (171 with diabetes and 70 without) aged $59.6 \pm 9.2$ years. The age of diabetic patients ranged between 45 and 86 years and 45 and 80 years for controls.

Table 1 shows the characteristics of the studied population. Data are presented according the presence of diabetes. Diabetic patients were older than controls 61.1 \pm 9.1 versus $55.7 \pm 8.5 ; \mathrm{p}<0.0001$. In term of morbidity, hypertension history was increased in diabetic patients in comparison with controls, $65 \%$ versus $30 \%$; $<0.0001$, and they were more frequently sick with higher number of comorbidities than controls $4.1 \pm 2.1$ versus $2.8 \pm 1.8$; $p$ $<0.0001$.

The prevalence of obesity was similar in the two groups with $63 \%$ in diabetics and $62 \%$ in controls.

To be noted, no significant difference was found between the two groups in terms of education levels (table I), nor in term of years of education which was found in diabetics and controls to be $6.34 \pm 5.885$ years and $7.57 \pm 6.051$ years; $p=0.220$, respectively. The percentage of illiterate, fundamental, intermediate and high education levels were 31, 28.5, 14.5 and $26 \%$ in diabetics and 26, 29, 14 and $31 \%$ in controls, respectively.

The two groups did not differ significantly in terms of other studied parameters.

Among diabetic patients, the mean $\mathrm{HbA1c}$ level was found to be $8.9 \pm 2.2 \%$ and the duration of diabetes was $13.8 \pm 9.7$ years. 
Table 1

Clinical and biological characteristics of the population

\begin{tabular}{lcccc}
\hline Characteristics & All & Diabetics & Controls & $\mathbf{p}^{*}$ \\
\hline Number & 241 & 171 & 70 & \\
Gender (Men $\%$ ) & $50 \%$ & $51 \%$ & $47 \%$ & 0,599 \\
Age (years) & $59,6 \pm 9,2$ & $61,1 \pm 9,1$ & $55,7 \pm 8,5$ & 0,000 \\
Marital status (married $\%$ ) & $84 \%$ & $82 \%$ & $89 \%$ & 0,230 \\
Level of Education (Illiterate $\%)$ & $29 \%$ & $31 \%$ & $26 \%$ & 0,786 \\
Past or current smoker & $40 \%$ & $39 \%$ & $44 \%$ & 0,414 \\
Hypertension history $(\%)$ & $55 \%$ & $65 \%$ & $30 \%$ & 0,000 \\
CHD (\%) & $23 \%$ & $25 \%$ & $17 \%$ & 0,202 \\
Stroke \& TIA (\%) & $10 \%$ & $11 \%$ & $6 \%$ & 0,195 \\
Depression (\%) & $9 \%$ & $7 \%$ & $13 \%$ & 0,145 \\
Comorbidities (number) & $3,7 \pm 2,1$ & $4,1 \pm 2,1$ & $2,8 \pm 1,8$ & 0,000 \\
BMI (kg/m ${ }^{2}$ ) & $32,0 \pm 6,7$ & $31,9 \pm 6,6$ & $32,3 \pm 7,0$ & 0,708 \\
WC (cm) & $110,8 \pm 14,2$ & $111,5 \pm 14,5$ & $109,2 \pm 13,4$ & 0,265 \\
GDS & $4,3 \pm 3,1$ & $4,3 \pm 3,0$ & $4,2 \pm 3,1$ & 0,878 \\
RUDAS & $25,67 \pm 2,80$ & $25,25 \pm 2,78$ & $26,71 \pm 2,57$ & 0,000 \\
MOCA & $19,97 \pm 5,25$ & $19,66 \pm 5,09$ & $20,66 \pm 5,58$ & 0,192 \\
\hline
\end{tabular}

Data are expressed as: Mean \pm SD and Percentage (\%), *: Probability of the Student's t-test (continuous variables) or Chi-Square test (categorical variables), CHD: coronary heart disease; TIA: transient ischemic attack; GDS: geriatric depression scale; WC: waist circumference; BMI: body mass index.

\section{Cognitive performance}

Both cognitive tests (RUDAS and MoCA) demonstrated a direct and significant correlation with the subjects' level of education $(\mathrm{r}=0.437, \mathrm{p}<0.0001$ and $\mathrm{r}=0.661, \mathrm{p}<0.0001)$ and BMI score $(\mathrm{r}=0.172, \mathrm{p}=0.009$ and $\mathrm{r}=0.139, \mathrm{p}=0.041)$, and an inverse relation with advancing age $(\mathrm{r}=-0.241 ; \mathrm{p}=0.0004$ and $\mathrm{r}=-0.162$; $\mathrm{p}=0.023$ ).

MoCA score was better in men $(18.8 \pm 5.5)$ than in women $(21.1 \pm 4.8) ; \mathrm{p}=0,001$, and also in married patients $(20.3 \pm 5.1)$ than in others $(20.3 \pm 5.1) ; p=0,016$, whereas no difference of RUDAS score was found in the distribution of gender or marital status.

Nevertheless, a highly significant correlation was observed between RUDAS and MoCA $(r=0,523$; $p<$ $0.000)$.

\section{Association between diabetes andcognitive function}

Diabetic patients are more likely to have less performance than controls in term of RUDAS score (Table 1 ), and this association persists after adjustment for age, gender, education level, GDS, BMI, hypertension history and number of comorbidities (26.7 \pm 0.31 in diabetics versus $25.3 \pm 0.19$ in controls; $p=0,0007$ ).

As we divided the RUDAS score into normal for these who scored $\geq 23$ and abnormal for those scoring less, we found that among diabetics $16 \%$ had abnormal scores compared to $3 \%$ for controls $(p=0.004)$.
Table 2

Analysis of factors associated with RUDAS score using multiple regression models

\begin{tabular}{lll}
\hline Source & $\begin{array}{l}\text { Regression } \\
\text { coefficient }\end{array}$ & $\mathbf{p}$ \\
\hline Intercept & $24.40 \pm 0.45$ & $<0.0001$ \\
Female gender & $-1.17 \pm 0.33$ & 0,0004 \\
Diabetes & $-1.21 \pm 0.34$ & 0,0005 \\
High level of education & $3.30 \pm 0.43$ & 0,0297 \\
& & \\
$\mathrm{R}^{2}$ & 0,2762 & \\
\hline
\end{tabular}

Regarding MoCA Scores, there was no significant difference between diabetics and controls (Table 1), as when MoCA was used as categorical score (abnormal if $<$ $26), 85 \%$ of the diabetics and $78 \%$ of the controls had abnormal results; $\mathrm{p}=0.194$.

In order to further investigate our primary finding that RUDAS score was worse in diabetics, additional multiple regression analyses were performed, adjusting for age, gender, education level, marital status, smoking, hypertension history, number of comorbidities, GDS, and BMI. The analyses show that the RUDAS scores decreased in females and with a history of diabetes, and increased when education level was high (Table 2). The model accounted for $28 \%$ of the total variance in RUDAS score.

Among diabetics, no statistically significant effect was foundfor neither the duration of diabetes on RUDAS $(\mathrm{r}=$ $-0,039 ; \mathrm{p}=0.607)$ or MoCA $(\mathrm{r}=-0,096 ; \mathrm{p}=0.235)$, nor for glycemic control (HbA1C) on RUDAS $(r=0,116 ; p=0.194)$ or MoCA ( $r=-0.027 ; p=0.776)$.

\section{Discussion}

The present case-control study conducted in a population sample from Saudi Arabia provides some elements of an effect of diabetes on cognition. Notably, the cognitive performance, as assessed by RUDAS, is poorer in diabetes, who are more likely to have cognitive impairment, than those who are not diabetics. This association was confirmed in multivariate analyses and shown to be independent of female gender and low education level all of which were associated with worse RUDAS cognitive score.

These results corroborate and extend results obtained in different populations reporting that diabetic patients are at an increased risk for development of cognitive performance or dementia $(4,13,14)$.

Although the design of the present study cannot provide an ascertain causality, it is of note that there are many direct mechanism linking diabetes and cognitive impairment such as insulin dysregulation (15-17) and chronic exposure to glucose (18-20). In the present study, we did not find a role for the duration of diabetes or glycemic control on cognitive performance. This was 
contrary to our expectation, but the observational study design with one assessment of $\mathrm{HbA} 1 \mathrm{C}$ does not allow ascertaining causality. Nevertheless, the landmark ACCORD MIND trial did not show a benefit for strict glycemic control on cognition as well (21).

In addition, the impact of the cardiovascular risk factors, which are increased in diabetic patients, could contribute to explain this association between diabetes and cognitive impairment (14). Indeed, several studies have indicated that individuals with cardiovascular risk factors and vascular alterations are at increased risk of developing cognitive disorders (22-24). In the present study, the prevalence of hypertension and the number of comorbidities were significantly higher in diabetic patient, but without significant direct association with cognitive performance as assessed by the both cognitive tests.

Our findings indicated a positive effect of increasing BMI on cognition (evident in both tests). This is in accordance with the results of some previous studies (25), but in contrast to others (26). However, the effect of BMI did not remain significant in the regression analyses (Table 2). Moreover, we have to mention that the prevalence of obesity is quite alarming in our sample. This however does reflect the unfortunate fact of the widespread of this problem in our society (1). Indeed, this high prevalence of obesity could make minimize the significance of the BMI when entered as covariable in the regression model.

As expected and shown in many previous studies (27, 28 ), cognitive tests scores herein were superior in those individuals that were more educated. Although education levels were similar in diabetics and controls in our study, it could be considered as a shared risk factor for cognitive impairment and diabetes since there is evidence that low educational levels increase the risk of diabetes (29) given the overall low education level among our study population. This last observation could contribute to explain our results demonstrating the cognitive impairment in diabetics using the RUDAS, but not the MOCA. Although MOCA is validated, quick, and easy to administer, it requires a certain level of education and therefore can lose its sensitivity in a low-education population. The MoCA was reported to be more sensitive in individuals with a high level of education (30), whereas the RUDAS has been found to be particularly helpful in populations with limited education $(31,32)$. Moreover, We have found RUDAS easy to administer, both for the testerand the tested, which is very important in an ethnically and educationally diverse population like ours. This also carries important implications infuture research in our region given the dearth of cognitive research in our region, perhaps due to the difficulty of administering the more popular cognitive tests.

Beyond diabetes and low education level, female gender was also independently associated with lower score son RUDAS. Indeed, it was reported that there is gender-difference in term of cognitive function in the developing countries (33). Since it was observed that the combining between socio-cultural factors with gender has more effect on cognition rather than sex alone (34), this negative effect of female gender on cognitive performance could also be explained by lesser levels of education in females who could for example lose their sex-advantage in terms of cardiovascular profile relating to sociocultural factors (35).

To our knowledge, this is the first study that addresses cognitive impairment in diabetics in an Arabic country. In addition, it is important to mention that our population included not only elderly but middle-aged as well as there is evidence of the role of mid-life rather than latelife diabetes in the development of cognitive impairment (36).

Our results could draw attention to this underrecognized problem of cognitive impairment that can lead to significant health and social outcomes, especially that diabetes in KSA is now a national health problem. Indeed, diabetics with cognitive impairment are more likely to experience poor compliance to medications, are more susceptible to medication errors, and less likely to measure blood sugars and adjust their medications properly. Moreover, the functional disability that could result from cognitive impairment adds up to the burden of diabetes on the person, caregivers, and the community as a whole.

Certain limitations in this study should be noted. The controlswere determined based on their self-reported health, raising the possibility of underreporting in the control group. Indeed, many reports highlight educational levels and ethnicity as biases in disease under-reporting in self-assessed health (37). Moreover, the observational study design does not allow ascertaining causality, and the cross sectional design does not allow testing for temporal changes in cognitive performance.

We believe that largerlongitudinal studiesare needed to furtheridentify possible modifiable risk factors for cognitive impairment in diabetes taking into account life style, education, contextual, social and metabolic proprieties of our population. Moreover, new cognitivemarkers such as advanced neuroimaging could be utilized to shed light on the possible underlying pathology and the potential relationship between diabetes and cognitive impairment.

However, in keeping with the present study results, diabetes and female gender increased the likelihood of classifying patients as cognitively impaired while high education level may protect them from cognitive impairment.

\section{Conclusion}

In our population with an alarming prevalence of obesity, diabetes was associated with poorer cognitive performance independent of female gender and low 
education levels, drawing attention to this underrecognized problem of cognitive impairment that can lead to significant health and social outcomes, particularly in areas with high prevalence of DM. RUDAS was found to be a very reasonable and convenient test to assess cognition in our sample characterized by a low educational level.

Ethical standards: The research was conducted in accordance withthe curren laws of KSA and obtained the approval from KAUH. The investigators undertook to respect the protocol in all respects.

Acknowledgment: The authors would like to extend their thanks to King Abdulaziz City for Science and technology (KACST) for the support of this research.

Conflict of interest: No

\section{References}

1. Al-Nozha MM, Al-Maatouq MA, Al-Mazrou YY, Al-Harthi SS, Arafah MR, Khalil MZ, et al. Diabetes mellitus in Saudi Arabia. Saudi medical journal. 2004;25(11):1603-10.

2. Alqurashi KA, Aljabri KS, Bokhari SA. Prevalence of diabetes mellitus in a Saudi community. Annals of Saudi medicine. 2011;31(1):19-23.

3. Federation ID. The global burden. IDF Diabetes Atlas. Sixth ed2013.

4. Mayeda ER, Whitmer RA, Yaffe K. Diabetes and cognition. Clinics in geriatric medicine. 2015;31(1):101-15, ix.

5. Cheng G, Huang C, Deng H, Wang H. Diabetes as a risk factor for dementia and mild cognitive impairment: a meta-analysis of longitudinal studies. Internal medicine journal. 2012;42(5):484-91.

6. Memish ZA, Jaber S, Mokdad AH, AlMazroa MA, Murray CJ, Al Rabeeah AA, et al. Burden of disease, injuries, and risk factors in the Kingdom of Saudi Arabia, 1990-2010. Preventing chronic disease. 2014;11:E169.

7. Bangen KJ, Gu Y, Gross AL, Schneider BC, Skinner JC, Benitez A, et al. Relationship Between Type 2 Diabetes Mellitus and Cognitive Change in a Multiethnic Elderly Cohort. Journal of the American Geriatrics Society. 2015;63(6):1075-83.

8. Nasreddine ZS, Phillips NA, Bedirian V, Charbonneau S, Whitehead V, Collin I, et al. The Montreal Cognitive Assessment, MoCA: a brief screening tool for mild cognitive impairment. Journal of the American Geriatrics Society. 2005;53(4):695-9.

9. Rowland JT, Basic D, Storey JE, Conforti DA. The Rowland Universal Dementia Assessment Scale (RUDAS) and the Folstein MMSE in a multicultural cohort of elderly persons. International psychogeriatrics / IPA. 2006;18(1):111-20.

10. Chaaya M, Sibai AM, Roueiheb ZE, Chemaitelly H, Chahine LM, Al-Amin H, et al. Validation of the Arabic version of the short Geriatric Depression Scale (GDS-15). International psychogeriatrics / IPA. 2008;20(3):571-81.

11. Iype T, Shaji SK, Balakrishnan A, Charles D, Varghese AA, Antony TP. Cognition in type 2 diabetes: Association with vascular risk factors, complications of diabetes and depression. Annals of Indian Academy of Neurology. 2009;12(1):25-7.

12. Basic D, Rowland JT, Conforti DA, Vrantsidis F, Hill K, LoGiudice D, et al. The validity of the Rowland Universal Dementia Assessment Scale (RUDAS) in a multicultural cohort of community-dwelling older persons with early dementia. Alzheimer disease and associated disorders. 2009;23(2):124-9.

13. Cukierman T, Gerstein HC, Williamson JD. Cognitive decline and dementia in diabetes--systematic overview of prospective observational studies. Diabetologia. 2005;48(12):2460-9.

14. Feinkohl I, Price JF, Strachan MW, Frier BM. The impact of diabetes on cognitive decline: potential vascular, metabolic, and psychosocial risk factors. Alzheimer's research \& therapy. 2015;7(1):46.

15. Craft S, Cholerton B, Baker LD. Insulin and Alzheimer's disease: untangling the web. Journal of Alzheimer's disease : JAD. 2013;33 Suppl 1:S263-75.

16. Moreira PI. Alzheimer's disease and diabetes: an integrative view of the role of mitochondria, oxidative stress, and insulin. Journal of Alzheimer's disease : JAD. 2012;30 Suppl 2:S199-215.

17. Ronnemaa E, Zethelius B, Sundelof J, Sundstrom J, Degerman-Gunnarsson $\mathrm{M}$, Berne $\mathrm{C}$, et al. Impaired insulin secretion increases the risk of Alzheimer disease. Neurology. 2008;71(14):1065-71.

18. Le Floch JP, Levy M, Mosnier-Pudar H, Nobels F, Laroche S, Gonbert S, et al Comparison of once- versus twice-daily administration of insulin detemir, used with mealtime insulin aspart, in basal-bolus therapy for type 1 diabetes: assessment of detemir administration in a progressive treat-to-target trial (ADAPT). Diabetes care. 2009;32(1):32-7.

19. Umegaki H, Kawamura T, Umemura T, Kawano N. Factors associated with cognitive decline in older adults with type 2 diabetes mellitus during a 6-year observation. Geriatrics \& gerontology international. 2015;15(3):302-10.

20. Exalto LG, Biessels GJ, Karter AJ, Huang ES, Quesenberry CP, Jr., Whitmer RA. Severe diabetic retinal disease and dementia risk in type 2 diabetes. Journal of Alzheimer's disease : JAD. 2014;42 Suppl 3:S109-17.

21. Launer LJ, Miller ME, Williamson JD, Lazar RM, Gerstein HC, Murray AM, et al. Effects of intensive glucose lowering on brain structure and function in people with type 2 diabetes (ACCORD MIND): a randomised open-label substudy. The Lancet Neurology. 2011;10(11):969-77.

22. Casserly I, Topol E. Convergence of atherosclerosis and Alzheimer's disease: inflammation, cholesterol, and misfolded proteins. Lancet. 2004;363(9415):1139-46.

23. Watfa G, Benetos A, Kearney-Schwartz A, Labat C, Gautier S, Hanon O, et al. Do Arterial Hemodynamic Parameters Predict Cognitive Decline Over a Period of 2 Years in Individuals Older Than 80 Years Living in Nursing Homes? The PARTAGE Study. Journal of the American Medical Directors Association. 2015;16(7):598-602.

24. Kaffashian S, Dugravot A, Elbaz A, Shipley MJ, Sabia S, Kivimaki M, et al. Predicting cognitive decline: a dementia risk score vs. the Framingham vascular risk scores. Neurology. 2013;80(14):1300-6.

25. Hughes TF, Borenstein AR, Schofield E, Wu Y, Larson EB. Association between late-life body mass index and dementia: The Kame Project. Neurology. 2009;72(20):1741-6.

26. Anstey KJ, Cherbuin N, Budge M, Young J. Body mass index in midlife and late-life as a risk factor for dementia: a meta-analysis of prospective studies. Obesity reviews : an official journal of the International Association for the Study of Obesity. 2011;12(5):e426-37.

27. Sharp ES, Gatz M. Relationship between education and dementia: an updated systematic review. Alzheimer disease and associated disorders. 2011;25(4):289-304.

28. Callahan CM, Hall KS, Hui SL, Musick BS, Unverzagt FW, Hendrie HC. Relationship of age, education, and occupation with dementia among a community-based sample of African Americans. Archives of neurology. 1996;53(2):134-40

29. Sacerdote C, Ricceri F, Rolandsson O, Baldi I, Chirlaque MD, Feskens E, et al. Lower educational level is a predictor of incident type 2 diabetes in European countries: the EPIC-InterAct study. International journal of epidemiology. 2012;41(4):1162-73.

30. Koski L, Xie H, Finch L. Measuring cognition in a geriatric outpatient clinic: Rasch analysis of the Montreal Cognitive Assessment. Journal of geriatric psychiatry and neurology. 2009;22(3):151-60.

31. Storey JE, Rowland JT, Basic D, Conforti DA, Dickson HG. The Rowland Universal Dementia Assessment Scale (RUDAS): a multicultural cognitive assessment scale. International psychogeriatrics / IPA. 2004;16(1):13-31.

32. Velayudhan L, Ryu SH, Raczek M, Philpot M, Lindesay J, Critchfield M, et al. Review of brief cognitive tests for patients with suspected dementia. International psychogeriatrics / IPA. 2014;26(8):1247-62.

33. Onadja Y, Atchessi N, Soura BA, Rossier C, Zunzunegui MV. Gender differences in cognitive impairment and mobility disability in old age: a crosssectional study in Ouagadougou, Burkina Faso. Archives of gerontology and geriatrics. 2013;57(3):311-8.

34. Ferreira L, Ferreira Santos-Galduroz R, Ferri CP, Fernandes Galduroz JC Rate of cognitive decline in relation to sex after 60 years-of-age: a systematic review. Geriatrics \& gerontology international. 2014;14(1):23-31.

35. Temmar M, Watfa G, Joly L, Kearney-Schwartz A, Youcef M, Bensalah S, et al. Elderly Algerian women lose their sex-advantage in terms of arterial stiffness and cardiovascular profile. Journal of hypertension. 2013;31(11):2244-50 discussion 50.

36. Rawlings AM, Sharrett AR, Schneider AL, Coresh J, Albert M, Couper D, et al. Diabetes in midlife and cognitive change over 20 years: a cohort study. Annals of internal medicine. 2014;161(11):785-93.

37. Mackenbach JP, Looman CW, van der Meer JB. Differences in the misreporting of chronic conditions, by level of education: the effect on inequalities in prevalence rates. American journal of public health. 1996;86(5):706-11. 